\title{
Modelling of solar granulation
}

\author{
H. J. Muthsam ${ }^{1}$, B. Löw-Baselli ${ }^{1}$, Chr. Obertscheider ${ }^{1}$, M. Langer ${ }^{1}$, \\ P. Lenz ${ }^{1,2}$ and F. Kupka ${ }^{3}$ \\ ${ }^{1}$ Institute of Mathematics, University of Vienna, Nordbergstrasse 15, A-1090 Vienna, Austria \\ email: herbert.muthsam@univie.ac.at \\ ${ }^{2}$ Institute for Astronomy, University of Vienna, Türkenschanzstrasse 17, \\ A-1180Vienna, Austria \\ ${ }^{3}$ Max-Planck-Institute for Astrophysics, Karl-Schwarzschild-Strasse 1, D-85741 Garching, \\ Germany
}

\begin{abstract}
We present the essential features of the ANTARES code. ANTARES has been developed to perform the simulation of astrophysical flows in one, two, or three dimensions. Using, in particular, the option of grid refinement, we present results for solar granulation achieved at very high spatial resolution.
\end{abstract}

Keywords. Sun: granulation, convection, hydrodynamics

\section{Introduction}

Modelling of solar granulation has now achieved a description of solar surface convection which is close to reality in many details, as shown by comparisons with observations, and an understanding of the basic physical processes involved (see e.g. Stein \& Nordlund 2000). Despite of that a number of important issues have remained unchecked. This concerns in particular processes occuring on small scales which neither observations nor models have resolved because of technical limitations.

Small scale features are, however, important because they may influence, via collective effects, the global outcome and the overall physical structure. In addition, recent observations show the existence of an important component, as of now not understood, of the solar magnetic field below the observational resolution limit (see, e.g., Lites \& Socas-Navarro 2004). This reinforces the interest in a study of small scales. We want to start therefore with high-resolution simulations of solar granulation. In order to commence with more simple problems and to figure out basic processes in the first place we consider here the radiation hydrodynamics case only (i.e. no magnetohydrodynamics), discuss mainly $2 \mathrm{D}$ models and make only a few remarks on the $3 \mathrm{D}$ case.

\section{The ANTARES code and the VIVAT package}

As a tool for investigations of solar granulation we have developed the ANTARES code (ANTARES = A Numerical Tool for Astrophysical RESearch). Its main features are

- in general: time-dependent compressible hydrodynamics and extensions (e.g., MHD) in 1D, 2D, 3D; Fortran90 code with a modular structure;

- numerics: conservation laws via three different high-resolution conservative schemes of the ENO (essentially non-oscillatory) type (see e.g. Liu \& Osher 1998), in principle of arbitrary high order even near discontinuities, in practice typically of order 6 in space and 3 in time; 
- radiative transfer: short characteristics method (Mihalas et al. 1978), interpolation of at least second order; frequency dependence accounted for by opacity binning (Nordlund 1982), typically with 1 (grey), 4 or 12 bins;

- gridding: logically rectangular; allows for grid-refinement in (logically) rectangular patches, even recursively; data from refinement are projected up to the coarser level;

- microphysics: realistic, i.e. OPAL(+Alexander), opacities and equation of state (Rogers et al. 1996, Alexander \& Ferguson 1994); if desired also in idealized form;

- parallelization: via MPI;

- portability: demonstrated by implementation on several architectures such as ○ AMD resp. Intel processors, Windows (Absoft, Compaq, Intel compilers);

- AMD resp. Intel processors, Linux (Absoft, Intel compilers);

- POWER processors, AIX (IBM Fortran compiler).

Analysis is performed via VIVAT (VIVAT = VIennese Visualization and Analysis Tool) which we are continuously extending. VIVAT is based upon the Visualization ToolKit VTK (http://www.vtk.org).

\section{Solar granulation in high resolution}

For the reasons explained in the introduction we use 2D models at first, starting with a relaxed $2 \mathrm{D}$ state, adopting grey opacities with realistic microphysics, overall therefore a semi-realistic model. Note that we are interested mainly in the development of downflowing plumes, i.e. in deeper layers, where the grey approximation is not unreasonable. The total size of the computational domain is $2.8 \times 11.2 \mathrm{Mm}^{2}$ (depth $\times$ width). Flux constancy is achived to within $1 \%$. This model has a spatially uniform $400 \times 1000$ grid, corresponding to a meshsize of $7 \times 11.2 \mathrm{~km}^{2}$. The refined subdomain has $648 \times 964$ gridpoints and a refinement factor of 4 in each direction; thus it encomprises $1.1 \times 2.7 \mathrm{Mm}^{2}$ on the sun with a meshsize of $1.75 \times 2.8 \mathrm{~km}^{2}$.

The high-resolution subdomain has been set to the region of a strong downdraft which existed already when the high-resolution option was switched on. Figure 1 shows this high-resolution part only a short time after high-resolution has been turned on, i.e. with the details of the low resolution run. In this short time the cool downflow channels have developed very sharp walls (in temperature or density).

Plumes can readily be investigated by examining temperature isohypses with a spacing of $1000 \mathrm{~K}$ or looking at density. In any such visualization procedure well defined cores of plumes are clearly visible. These cores are embedded within considerably broader downflow patterns (actually not always a downflow because the plumes are frequently deflected sideways or even upwards). Our main results from this run are:

- in high-resolution runs plumes split more easily into finer ones which remain, however, connected to the original plume.

- In high-resolution runs the diameter of the cores of the plumes may well measure only $25 \mathrm{~km}$ or even less, quite at even the limits of our high-resolution runs. There are hints that especially near strong curvature instabilities set in which can possibly tear plumes into parts, altering the basic topology of hitherto connected flows, when investigations were conducted in still higher resolution. We are working on such extra high-resolution models.

- In the presently used high resolution, anyway, even the very narrow plumes are surprisingly stable; if occasionally considerably cooler, denser material rushes down an already existing channel, as it occurs, this material follows the path of preexisting channels astonishingly faithfully as much as we have seen up to now. 
Note that we have applied high-resolution to an already preexisting plume. The question about what happens, if high-resolution is applied to a newly evolving plume with its head plunging violently down into the more or less undisturbed layers, is presently under investigation.

Turning briefly to the $3 \mathrm{D}$ case we remark that a similar simulation with grid refinement (fine meshsize $15 \times 22 \times 22 \mathrm{~km}^{3}$ ) reveals a remarkably smooth downflow (i.e. the isohypses of various quantities are not rugged), albeit with steep horizontal gradients.

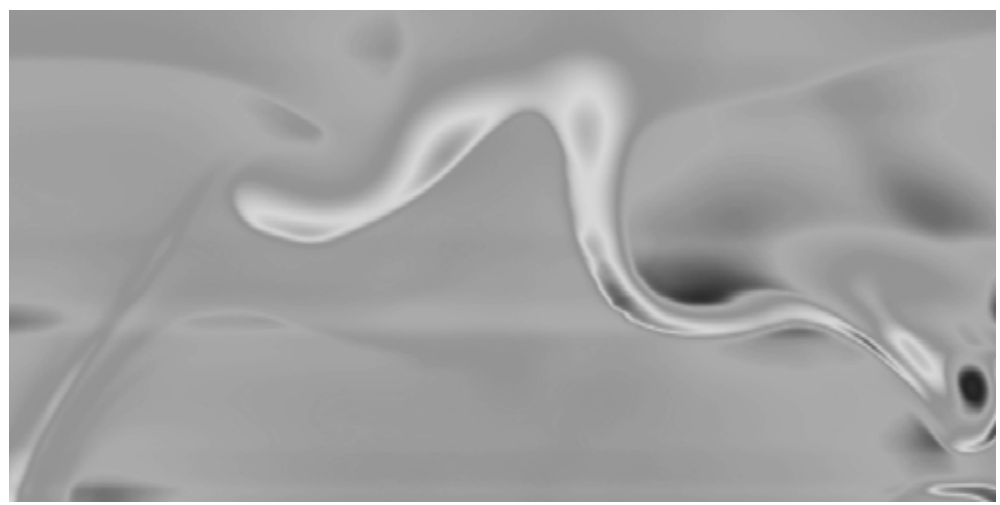

(a)

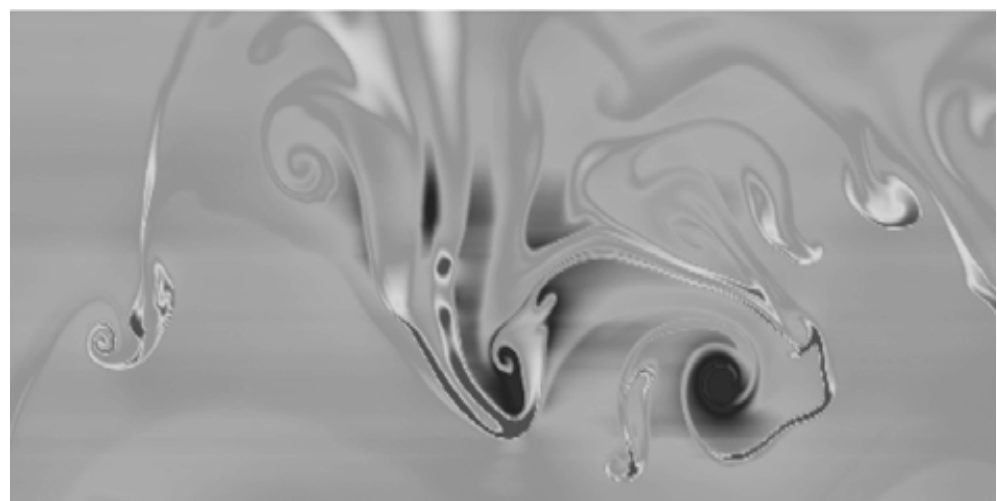

(b)

Figure 1. Density with horizontal average subtracted. (a) initial state from the low resolution run; (b): high resolution features have developed many of which are not seen if the low resolution run is conducted up to that point of time.

\section{Acknowledgement}

We gratefully acknowledge a grant from the Austrian Science Fund (project AP17024).

\section{References}

Alexander, D.R. \& Ferguson, J.W. 1994, ApJ 437, 979

Lites, B.W. \& Socas-Navarro, H. 2004, ApJ 613, 600

Liu, X.-D. \& Osher, S. 1998, JCP 142, 304

Mihalas, D., Auer, L.H. \& Mihalas, B.W. 1978, ApJ 220, 1001

Nordlund, A. 1982, A\&SA 107, 1

Rogers, F.J., Swenson, F.J. \& Iglesias, C.A. 1996, ApJ 456, 902

Stein, R.F. \& Nordlund, Å. 2000, Solar Phys. 192, 91 